IFUP-TH 3/93

hep-th/9301067

\title{
A Generalized Uncertainty Principle in Quantum Gravity
}

\author{
Michele Maggiore \\ I.N.F.N. and Dipartimento di Fisica dell'Università, \\ piazza Torricelli 2, I-56100 Pisa, Italy.
}

\begin{abstract}
We discuss a Gedanken experiment for the measurement of the area of the apparent horizon of a black hole in quantum gravity. Using rather general and model-independent considerations we find a generalized uncertainty principle which agrees with a similar result obtained in the framework of string theories. The result indicates that a minimum length of the order of the Planck length emerges naturally from any quantum theory of gravity, and that the concept of black hole is not operationally defined if the mass is smaller than the Planck mass.
\end{abstract}


1. In field theories which do not involve gravitation the transition from a classical to a quantum description is performed 'superimposing' a quantum structure (e.g., commutation relations) to the classical theory; when we attempt to combine gravitation and quantum theory, however, we expect that at distances of the order of the Planck length the very notion of space-time might need a radical revision. Because of this, one cannot exclude a priori that a proper quantum theory of gravity might require a modification of basic quantum principles.

In this Letter we examine the uncertainty principle and suggest that in quantum gravity it is indeed modified. This result, in itself, is not new; a generalized uncertainty principle has already been proposed in the context of string theories in refs. [1-2] through an analysis of Gedanken string collisions at planckian energies [2-4] (for a recent review, see [5]), and in [6] through a renormalization group analysis applied to the string. Our new point is that we do not consider strings, but use only general model-independent properties of a quantum theory of gravitation: our main physical ingredient is the Hawking radiation [7]. The functional form of the generalized uncertainty principle that we obtain agrees with the one found in string theory.

Let us consider a Reissner-Nordström black hole with mass $M$ and charge $Q$ (the generalization of our considerations to Kerr-Newman black holes does not present conceptual difficulties). The apparent horizon is defined [B] as the outer boundary of a region of closed trapped surfaces; it has spherical topology and, in Boyer-Lindquist coordinates, it is located at $r=R_{h}$,

$$
R_{h}=G M\left[1+\left(1-\frac{Q^{2}}{G M^{2}}\right)^{1 / 2}\right] .
$$

(We set $c=1$ but write explicitly $\hbar$ and $G$ in the following). In classical general relativity an observer has no direct access to the apparent horizon: no signal is emitted from the black hole. If an observer wants to obtain the area of the apparent horizon, he should measure the mass and charge of the black hole from the motion of test particles at infinity, and then resort to the theory, which predicts the relation (1) for $R_{h}$ as a function of $M$ and $Q$; the area of the horizon is then $A=4 \pi R_{h}^{2}$. Alternatively, one can perform a scattering experiment and again resort to the theory which predicts the relation between the measured cross section and $R_{h}$. For instance, for ultra-relativistic particles impinging on a Schwarzschild black hole, general relativity predicts a capture cross section $\sigma=(27 / 4) \pi R_{h}^{2}$. Instead, it is 
not possible to measure the area of the horizon directly, that is, using an apparatus which records photons emitted by the horizon itself, and without having to resort to relationships predicted by the theory.

In this sense, from an operational point of view, in classical general relativity eq. (1) must be considered as a definition of $R_{h}$, rather than a prediction which can be tested experimentally.

In a quantum theory of gravitation, however, the emission of Hawking radiation allows an observer at infinity to receive a signal coming from the apparent horizon, and therefore to perform a direct measurement of its area, at least at the level of a Gedanken experiment. The radius of the horizon and the mass and charge of the black hole become three quantities subject to independent experimental determination. It then makes sense to ask whether the relation given in eq. (1) is satisfied. In the following we examine whether there is an intrinsic limitation to the precision of the experimental determination of $R_{h}$.

First, let us better specify the setting of our Gedanken experiment. In the case of a Schwarzschild black hole $(Q=0)$ the Hawking radiation is emitted spontaneously. Instead, an extremal $\left(Q^{2}=G M^{2}\right)$ Reissner-Nordström black hole has zero temperature. In particular, one can consider a magnetically (rather than electrically) charged black hole in a world with no magnetically charged particles. In this case the black hole cannot discharge through pair creation [9], and it is expected to be stable, both classically and quantum mechanically. In order to measure directly the area of its apparent horizon we can consider an experiment similar to the Heisenberg microscope (fig. 1): a photon with wavelength $\lambda$ is sent from infinity and absorbed by the black hole. After absorption, the black hole has a mass $M+\Delta M$, with $\Delta M=h / \lambda$, and it is not extremal. One expects that it will decay back to the extremal state. The emission spectrum in general will not consist of thermal Hawking radiation, since a thermal description breaks down for near-extremal black holes [11]. It might even consist of a single photon, again of wavelength $\lambda$. For definiteness, we indeed consider the situation in which a single photon is emitted. If the black hole rather decays with emission of many particles (not

\footnotetext{
${ }^{1}$ We ignore the (classically forbidden) possibility that the black hole may break apart into smaller extremal black holes. At the quantum level, this possibility has been suggested recently [10. However the existence of such processes, if triggered by the incident photon, would only increase the disturbance induced by the measurement, and therefore can only strengthen the uncertainty principle that we will find.
} 
necessarily photons, of course), our considerations apply separately to each of them, and our conclusions are unchanged. At 90 degrees with the incoming photon, a microscope detects the photon emitted (fig. 1). Repeating the experiment and recording many photons, we obtain an 'image' of the black hole. Togheter with a measurement of the distance $d$ of the black hole from the microscope, this provides a measurement of $R_{h}$. The distance $d$ can be measured using again the Hawking radiation. One can (in principle) measure the direction of propagation of photons emitted at different angles and trace them back in order to locate the position of the center of the black hole.

Which experimental precision can be obtained in this measurement of the radius of the horizon? Of course, a photon with wavelength $\lambda$ cannot carry information on a more detailed scale than $\lambda$ itself. As in the classical Heisenberg analysis, the resolving power of the microscope gives a minimum error, $\Delta x^{(1)}$, on $R_{h}$,

$$
\Delta x^{(1)} \sim \frac{\lambda}{\sin \theta},
$$

where $\theta$ is the angle defined in fig. 1 . Since the error in the final momentum of the black hole is $\Delta p \sim h \sin \theta / \lambda$, this gives the standard Heisenberg relation, $\Delta x^{(1)} \sim \hbar / \Delta p$. More precisely, from infinity we are actually observing the projection of the black hole on a $(x, y)$-plane parallel to the microscope (see fig. 1); $\Delta x^{(1)}$ is the error on the radius of the horizon measured along the $x$-direction and $\Delta p$ the error on the final black hole momentum along the same direction.

Besides, during the emission process the mass of the black hole varies from $M+\Delta M$ to $M$, and the radius of the horizon changes accordingly.2' At the moment of the measure, that is, when the light quantum is being emitted by the black hole, the quantity that we are measuring changes discontinuosly. It does not make sense to ask whether the information carried by the outgoing photon refers to the black hole immediately before emission, or immediately after, or to something in between. The corresponding error must be considered as intrinsic to the measurement. This gives a second source of error on $R_{h}$ which, for a Schwarzschild black hole, is $\Delta x^{(2)} \sim 2 G \Delta M$ and for

\footnotetext{
${ }^{2}$ We neglect the variation in angular momentum in the absorption/emission process. It does not alter the final result.
} 
a general Reissner-Nordström black hole is

$$
\Delta x^{(2)} \sim G \Delta M+\sqrt{(G M+G \Delta M)^{2}-G Q^{2}}-\sqrt{(G M)^{2}-G Q^{2}} \geq 2 G \Delta M .
$$

Since $\Delta M=h / \lambda$, we get an uncertainty

$$
\Delta x^{(2)} \sim \frac{\hbar G}{\lambda}=\frac{L_{\mathrm{Pl}}^{2}}{\lambda},
$$

where $L_{\mathrm{Pl}}$ is the Planck length. Note that $\Delta x^{(2)}$ is only a lower bound on the uncertainty, and only a Schwarzschild black hole can saturate it. For instance, for an extremally charged black hole with $M \gg \Delta M$, eq. (3) gives $\Delta x^{(2)} \simeq G \sqrt{2 M \Delta M} \gg 2 G \Delta M$.

If we combine $\Delta x^{(1)}$ and $\Delta x^{(2)}$ linearly (this point will be discussed in more detail below) and use the trivial inequality

$$
\frac{\lambda}{\sin \theta} \geq \lambda
$$

we get

$$
\Delta x \gtrsim \lambda+\text { const. } \frac{L_{\mathrm{Pl}}^{2}}{\lambda} .
$$

In our approach the relative numerical constant between the two terms cannot be predicted. Indeed, in order to write a relation, like eq. (6), which does not depend on the features of the apparatus, we have eliminated $\theta$ using the trivial inequality, eq. (5); the price we pay is that we cannot consistently estimate the value of this constant (more on this below).

Eq. (6) implies that there exists a minimum error $\left.(\Delta x)\right|_{\min } \sim$ const. $L_{\mathrm{Pl}}$. Therefore the statement that the radius of the horizon is equal to the right hand side of eq. (1) has no operational meaning if we aim at a precision better that $L_{\mathrm{Pl}}$.

It is also suggestive to write a lower bound on $\Delta x$ in terms of $\Delta p \sim$ $h \sin \theta / \lambda$. In this case, $\Delta x^{(1)} \sim \hbar / \Delta p$. As for $\Delta x^{(2)}$, remember that eq. (幽) is due to the discontinuous change of the horizon during the measurement, and it is not directly related to the uncertainty in the (x-component) of the black hole momentum after the measure; in fact, it is present even when the latter is zero (that is, in the limit $\theta \rightarrow 0$ ). However, we can use the trivial inequality $\Delta p / \sin \theta \geq \Delta p$ to obtain

$$
\Delta x \gtrsim \frac{\hbar}{\Delta p}+c G \Delta p
$$


again with a relative constant $c$ which our model-independent arguments cannot predict.

2. It is natural to investigate whether the relation given in eq. (7), which has been obtained considering only a very specific measurement, has a more general validity in quantum gravity. A definite answer to this question presumably cannot be given using only the rather general and modelindependent arguments that we have presented, and one should consider the problem within a specific theory -e.g., string theory. We see two natural interpretations of eq. (77). One, more restrictive, is that it expresses nothing more than the fact that, because of quantum fluctuations, the concept of horizon in quantum gravity becomes uncertain, and is not defined on a scale smaller than $L_{\mathrm{Pl}}$. This fact, in itself, is not very surprising: it has already been noted (see e.g. [12]) that if $M \sim M_{\mathrm{Pl}}$ (where $M_{\mathrm{Pl}}$ is the Planck mass) the Compton radius of the black hole approaches its Schwarzschild radius, and quantum fluctuations in the black hole position affects the definition of the horizon. Our result, however, is somewhat stronger; we have shown that the concept of horizon is not defined, operationally, at scales smaller than $L_{\mathrm{Pl}}$; this implies that it does not make sense to talk of "black holes" with $M<M_{\mathrm{Pl}}$, nor to expect that, for $M \ll M_{\mathrm{Pl}}$ (and therefore for elementary particles) some sort of gravitational substructure, suitably modified by quantum effects, exists at scales of order of the Schwarzschild radius. It is more appropriate to consider the Compton radius $\hbar / M$ and the Schwarzschild radius $2 G M$ as mutually exclusive attributes of an object of mass $M$, which make sense only in the limits $M \ll M_{\mathrm{Pl}}$ and $M \gg M_{\mathrm{Pl}}$, respectively.

A second possible interpretation of the result that we have found is that it is an example of a more general situation, and that eq. (7) is indeed a generalized uncertainty principle which governs all measurement processes in quantum gravity. This possibility seems to us particularly attractive, for at least three reasons: 1) it implies that a minimal observable length emerges naturally from a quantum theory of gravity. 2) Eq. (7) is independent of the mass and charge of the black hole and, so to say, has lost any memory of the particular measurement which was analyzed. We have seen that in general $\Delta x^{(2)}$ depends on the mass and charge of the black hole, see eq. (3); it is non trivial that in the $(M, Q)$-plane it has a minimum, non zero, value. 3) Eq. (7) agrees with the result found in string theory [1, 2, 6]. Notice that, while in string theory the linear dependence of $\Delta x^{(2)}$ on $\Delta p$ comes out 
after a rather technical analysis, in our picture it is a simple consequence of the linear dependence of the Schwarzschild radius on the mass. The string theory analysis, on the other hand, can also predict (the order of magnitude of) the constant $c$ in eq. (7), and the result is $c \sim \alpha^{\prime}$, where $\alpha^{\prime}$ is the string tension (in Planck units). It is clear that such a result cannot be obtained with the general, model-independent, considerations that we have presented; from this point of view, it is welcome that eqs. (6,7) could only be obtained using the inequality $\sin \theta \leq 1$ which, on the one hand, allows us to get rid of the dependence on the apparatus, but on the other hand forbids us to give a consistent estimate of the numerical constant in eqs. (6.,7); otherwise, it is clear that we would have necessarily obtained a result of order one. We can state, however, that this constant is larger than a number of order one (and hence non-zero), again in agreement with the string prediction.

Notice further that the pointlike limit is obtained in string theory letting $\alpha^{\prime} \rightarrow 0$. In this limit the term linear in $\Delta p$, in the generalized uncertainty principle predicted from strings, disappears. Since however we have found, on rather general grounds, that this term is indeed present, one could take our result as an indication of the fact that it is not possible to construct a consistent quantum theory of gravity with pointlike objects. The same result is suggested by the emergence of a minimum measurable distance.

In eqs. (6.7), for the sake of definiteness, we have combined $\Delta x^{(1)}$ and $\Delta x^{(2)}$ linearly. More in general, it is suggestive to introduce a function $R_{*}(M)$, which in the proper limits reduces respectively to the Compton radius $R_{C}=\hbar / M$ and to the Schwarzschild radius $R_{S}=2 G M$

$$
R_{*}(M) \rightarrow \begin{cases}\hbar / M & \text { if } M \ll M_{\mathrm{Pl}} \\ 2 G M & \text { if } M \gg M_{\mathrm{Pl}}\end{cases}
$$

and which reaches a minimum value $\left.\left(R_{*}\right)\right|_{\min } \sim$ const. $L_{\mathrm{Pl}}$ when $M \sim$ const. $M_{\mathrm{Pl}}$. Loosely speaking, $R_{*}$ interpolates between the elementary particle regime $\left(M \ll M_{\mathrm{Pl}}\right)$, where an object with mass $M$ has a characteristic length $\sim R_{C}$ and the black hole regime $\left(M \gg M_{\mathrm{Pl}}\right)$, with characteristic length $\sim R_{S}$. It is tempting to interprete this function as the typical length associated with an object of mass $M$. In particular, this would illustrate the mutually exclusive nature of $R_{C}$ and $R_{S}$ mentioned above; these quantities would correspond to different limits of the same function; so that it does not make sense to talk of the Compton radius of a black hole, or of the Schwarzschild radius of an elementary particle. 
In terms of this function, the generalized uncertainty principle reads

$$
\Delta x \geq R_{*}(\Delta p) .
$$

Of course, both a confirmation of the interpretation of $R_{*}$ and its precise form can only be obtained by a full quantum theory of gravity.

I thank A. Di Giacomo, K. Konishi, M. Mintchev and G. Paffuti for useful comments.

\section{References}

[1] G. Veneziano, Europhys. Lett. 2 (1986) 199; Proc. of Texas Superstring Workshop (1989).

D. Gross, Proc. of ICHEP, Munich (1988).

[2] D. Amati, M. Ciafaloni and G. Veneziano, Phys. Lett. B216 (1989) 41.

[3] D. Amati, M. Ciafaloni and G. Veneziano, Phys. Lett. B197 (1987) 81; Int. J. Mod. Phys. A3 (1988) 1615; Nucl. Phys. B347 (1990) 530.

[4] D.J. Gross and P.F. Mende, Phys. Lett. B197 (1987) 129; Nucl. Phys. B303 (1988) 407.

[5] M. Ciafaloni, 'Planckian Scattering beyond the Eikonal Approximation', preprint DFF 172/9/'92 (1992).

[6] K. Konishi, G. Paffuti and P. Provero, Phys. Lett. B234 (1990) 276.

[7] S.W. Hawking, Comm. Math. Phys. 43 (1975) 199.

[8] S.W. Hawking and G.F.R. Ellis, The large scale structure of space-time, Cambridge University Press, (1973) Cambridge.

[9] G.W. Gibbons, Comm. Math. Phys. 44 (1975) 245.

[10] D. Brill, Phys. Rev. D46 (1992) 1560.

J.H Horne and G.T. Horowitz, "Black Holes Coupled to a Massive Dilaton", preprint UCSBTH-92-17, hep-th/9210012. 
[11] J. Preskill, P. Schwarz, A. Shapere, S. Trivedi and F. Wilczek, Mod. Phys. Lett. A6 (1991) 2353.

[12] S. Coleman, J. Preskill and F. Wilczek, Nucl. Phys. B378 (1992) 175. 


\section{FIGURE CAPTION}

Fig. 1: The Heisenberg microscope experiment described in the text. A photon moving along the positive $x$-axis is absorbed by an extremal black hole; a microscope detects the induced Hawking radiation, at a distance $d$ along the $z$-axis; $\theta$ is the angular opening of the microscope. The projection of the black hole onto the $(x, y)$-plane is a circle whose radius is measured in the experiment. 